livraisons

d'Histoire

de l'Architecture

\section{Livraisons de l'histoire de l'architecture}

$21 \mid 2011$

Bâtir et orner

\title{
Quelques fleurons des fonds Baltard (332 AP) et Destailleur (536 AP)
}

The Baltard and Destailleur funds, archive jewels

Meisterstücke aus den Nachlässen Baltard (332 AP) und Destailleur (536 AP)

Jean-Charles Cappronnier

\section{(2) OpenEdition}

Journals

Édition électronique

URL : http://journals.openedition.org/lha/275

DOI : 10.4000/lha. 275

ISSN : 1960-5994

Éditeur

Association Livraisons d'histoire de l'architecture - LHA

Édition imprimée

Date de publication : 10 juin 2011

Pagination : 63-78

ISSN : 1627-4970

Référence électronique

Jean-Charles Cappronnier, "Quelques fleurons des fonds Baltard (332 AP) et Destailleur (536 AP) », Livraisons de l'histoire de l'architecture [En ligne], 21 | 2011, mis en ligne le 10 juin 2013, consulté le 19 avril 2019. URL : http://journals.openedition.org//ha/275; DOI : 10.4000//ha.275

Ce document a été généré automatiquement le 19 avril 2019

Tous droits réservés à l'Association LHA 


\title{
Quelques fleurons des fonds Baltard (332 AP) et Destailleur (536 AP)
}

\author{
The Baltard and Destailleur funds, archive jewels \\ Meisterstücke aus den Nachlässen Baltard (332 AP) und Destailleur (536 AP)
}

\author{
Jean-Charles Cappronnier
}

1 Parmi les vingt-cinq fonds privés d'archives d'architecte conservés aux Archives nationales de Paris, douze concernent, en totalité ou en partie, le XIX ${ }^{e}$ siècle. Depuis les papiers de Pierre-François-Léonard Fontaine (1762-1853) jusqu'au fonds des architectes Jean Bélizaire (1828-1899) et René Moreau (1858-1924), architectes des Monuments historiques à Moulins, c'est toute la continuité du siècle et la diversité de sa production architecturale qui est envisagée et représentée au sein de ces archives. Archives néanmoins très inégalement conservées, tant par leur volume que par leur teneur. Entre l'unique carton rassemblant des pièces se rapportant à la production de François Bélanger (1745-1818), Jean-Baptiste Lepère (1761-1844) et Jacques-Ignace Hittorff (1793-1867) et la centaine de cartons et portefeuilles des archives Destailleur, l'amplitude est, en effet, considérable. De même certains fonds mêlent, de façon parfois inextricable, papiers familiaux et papiers d'agence. Le fonds d'Henri (1801-1875) et de Léon Labrouste (1846-1904), réuni par ce dernier, rassemble, pour l'essentiel, des archives à caractère généalogique et familial regroupées par Léon pour composer une histoire de la famille, mais viennent s'y intercaler des papiers se rapportant à la carrière et à l'œuvre d'Henri et de Théodore (1799-1885) Labrouste. Les archives ont pu également subir des tris drastiques émanant des praticiens eux-mêmes ou de leurs ayant-droits. Enfin, corollaire de cette dernière constatation, bon nombre de ces archives ont perdu la totalité ou la quasi-totalité de leurs pièces écrites : c'est le cas, en particulier, des archives d'Auguste (1816-1885) et Lucien (1849-1916) Magne, de celles de Charles Girault (1851-1933) ou encore du fonds Destailleur.

2 Les papiers Baltard et Destailleur, constituent, avec les archives Sanson, les fonds les plus riches et sans doute les mieux conservés parmi ceux donnés ou déposés aux Archives nationales à Paris. Si les archives de la dynastie Ernest (1836-1918), Maurice (1864-1917) 
et Jean (1895-1971) Sanson sont bien connues, notamment, par l'étude des réalisations du fondateur de la dynastie, deux de ses œuvres les plus célèbres étant la restauration du château de Chaumont-sur-Loire et l'hôtel de Castellane à Paris - plus connu sous le nom de Palais rose -, les fonds Baltard et Destailleur, tout aussi prolixes mais très différents dans leur composition et leur organisation archivistiques, présentent le plus haut intérêt aux titres de l'histoire et de l'histoire de l'art.

\section{Les archives de la dynastie Baltard : Louis-Pierre (1764-1846) et Victor (1805-1874)}

3 Le dépôt, devenu don, des archives Baltard aux Archives nationales à Paris, est le fruit d'une initiative de l'un des descendants de ces architectes, l'abbé Duval-Arnould, en 1973. C'est d'ailleurs la famille Arnould, issue de la fille unique de Victor Baltard, qui avait hérité de la villégiature familiale de Sceaux et des archives. Celles-ci ont été profondément réorganisées et remodelées par la famille, sans doute à la fin du XIX ${ }^{\mathrm{e}}$ siècle et au début du $\mathrm{XX}^{\mathrm{e}}$ siècle, comme en témoignent les conditionnements utilisés pour certaines pièces (pochettes de réemplois provenant de périodiques). Il en résulte un classement globalement thématique, réparti entre cartons et portefeuilles de pièces isolées en fonction du format de celles-ci. Ce classement, qui révèle, par ailleurs, de très importantes lacunes, a sans nul doute profondément bouleversé l'organisation originelle de ces archives où se croisent et s'entrecroisent papiers familiaux et dossiers d'affaires. De surcroît, et contrairement à bon nombre d'autres fonds d'archives d'architectes de cette époque, ce sont les pièces écrites qui y dominent, au détriment des documents figurés, souvent isolés ou réduits à quelques dossiers peu abondants numériquement.

4 La part des documents familiaux y est importante, au-delà des simples pièces à caractère généalogique. On y relève, outre une volumineuse correspondance qui, aussi bien sur le plan personnel que professionnel, constitue l'une des ressources les plus précieuses de ce fonds, d'abondants matériaux relatifs à la vie familiale du père, Louis-Pierre et du fils, Victor. On peut ainsi mentionner l'existence du journal de Mme Debrasseur, belle-mère de Louis-Pierre, tenu au tournant des XVIII e et XIX ${ }^{e}$ siècles. On peut encore citer les correspondances échangées entre Victor et Adeline Lequeux, son épouse, ou encore le registre de visites ouvert lors de la maladie de Victor, à l'extrême fin de 1873, maladie qui devait l'emporter au début de l'année suivante.

5 Si la formation de Louis-Pierre est peu représentée dans ce fonds, en revanche celle de Victor, et, en particulier, son séjour à Rome, entre 1833-1838, y est abondamment illustrée, en premier lieu par la correspondance reçue de sa famille et de ses amis condisciples ou non de la villa Médicis -, que ce soit les architectes Garrez ou Morey ou que ce soit le peintre Hippolyte Flandrin et, en second lieu, par de nombreux dessins ou croquis exécutés lors de son séjour romain et des voyages effectués dans les Pouilles et en Sicile.

Quant aux dossiers de projets, il est parfois délicat d'établir un lien organique entre le classement typologique - édifices civils, religieux - et individualisé (rarement plus de cinq pièces) que nous offrent les portefeuilles et l'organisation approximativement thématique que revêtent les cartons. L'œuvre construite de Louis-Pierre, peu importante numériquement au regard de ses nombreux projets non réalisés ou de ses participations infructueuses à des concours (ill. 1), y est essentiellement dévoilée par un riche dossier se 
rapportant à la construction du palais de justice de Lyon avec sa prison, engagée en 1835, à la suite d'un concours organisé dès 1827 et où figurent, fait rarissime le concernant, des dessins d'agence structurés par une nomenclature.

III. 1 : projet de monument à la gloire des armées de la République, à ériger sur la place de la Concorde

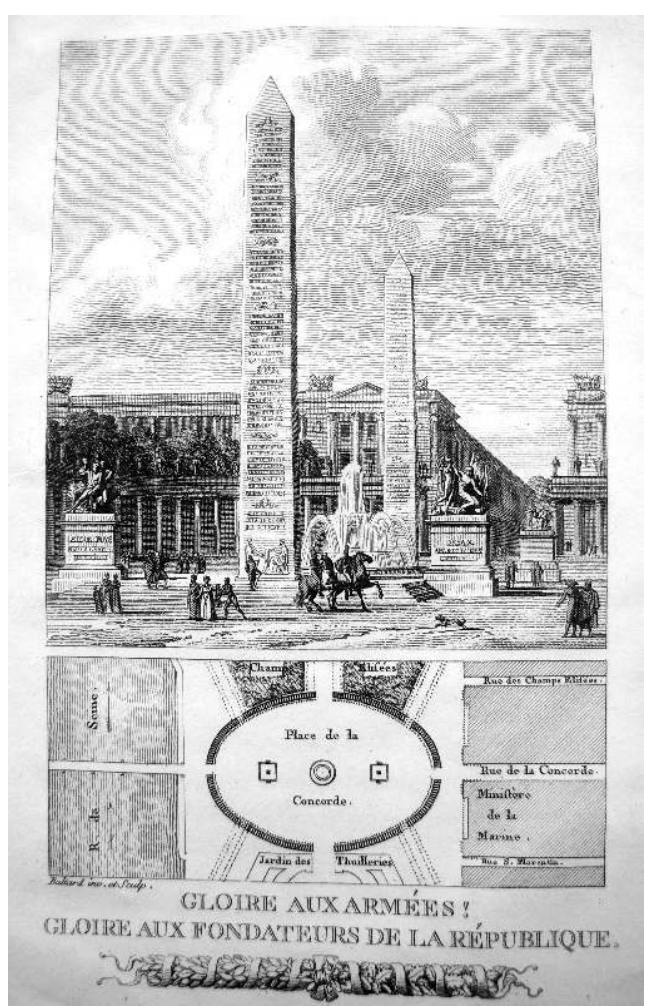

Plan et élévation, dessinés et gravés par Louis-Pierre Baltard, [1800]. Arch. nat., 332/AP/1.

Cl. de l'auteur.

7 Le fonds d'archives témoigne de la multiplicité des projets qui suivent les aléas des contraintes locales avec, de surcroît, de longues hésitations sur le choix de l'emplacement assigné au nouveau palais de justice (ill. 2). 
III. 2 : Projet pour l'établissement du palais de justice et d'une maison d'arrêt semi-panoptique, dans l'île de la Saône, aux Roches

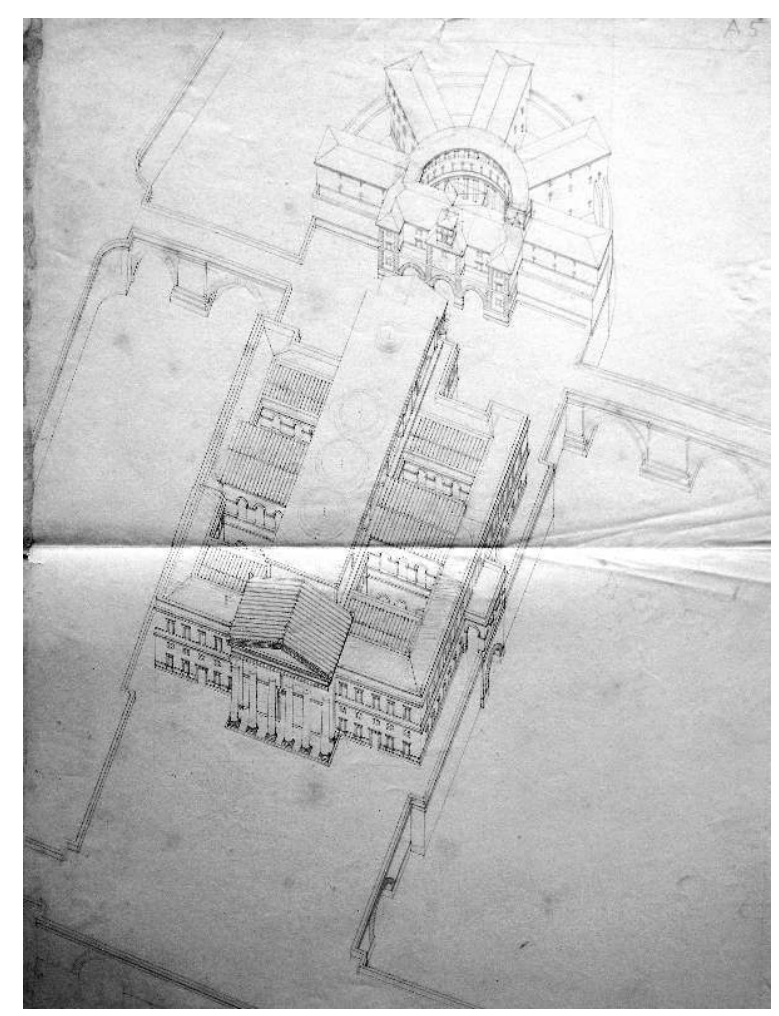

Perspective axonométrique [par Louis-Pierre Baltard, 1829-1830]). Arch. nat., 332/AP/22.

Cl. de l'auteur.

8 Hormis ce projet, on relève un certain nombre de plans et de pièces écrites relatifs au projet du Palais de Justice de Largentière, non réalisé par Baltard, mais sur des plans de l'architecte Weil fortement inspirés de ceux de ce dernier établis en 1827. Nommé architecte des prisons du département de la Seine, puis du Panthéon, par le préfet Chabrol en 1813, Louis-Pierre Baltard lègue avec ses archives, un exemplaire de son célèbre ouvrage, L'Architectonographie des prisons (1821), ainsi que mémoire manuscrit et rapports sur le même sujet. Enfin, quelques projets ponctuels ne sont documentés que par de rares dessins, comme la fontaine du marché Saint-Honoré à Paris (1834). 


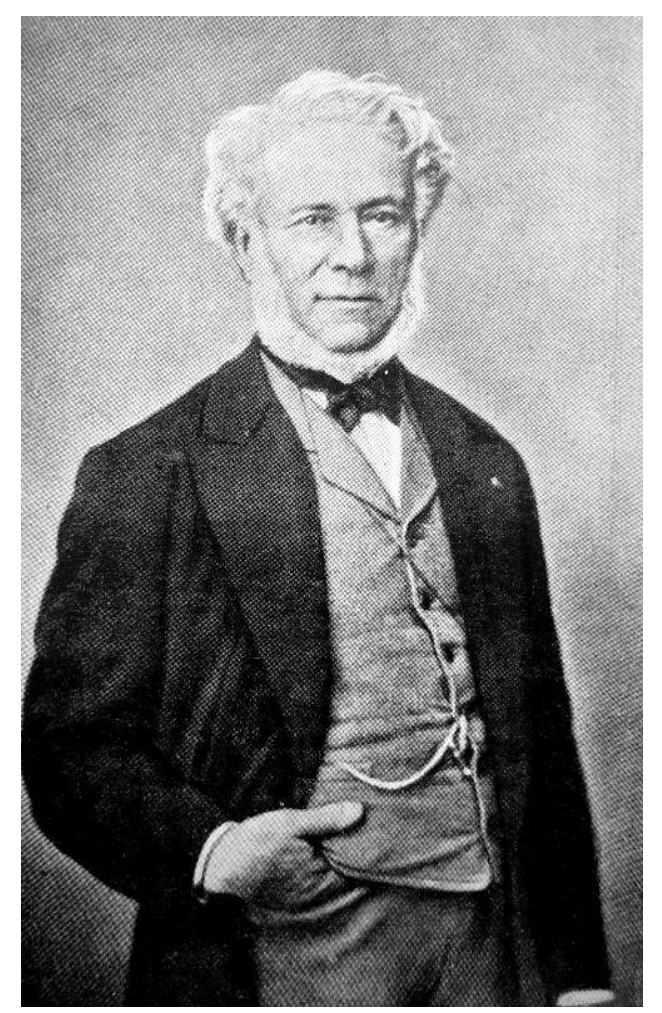

Épreuve photographique non datée, [v. 1860], reproduite dans la brochure Victor Baltard, 1805-1874, par Louis Arnould, 1939). Arch. nat., 332/AP/2.

Cl. de l'auteur.

9 Victor Baltard (ill. 3) a exercé, on le sait, dans le sillage de son père, d'éminentes fonctions au sein de l'administration du département de la Seine. Nommé, quelque temps après son retour de Rome, inspecteur des travaux d'art de la Ville de Paris pour ce qui concerne les édifices religieux, en 1841, charge qu'il exerce jusqu'en 1860, il est également architecte diocésain «du département de la Seine» [sic], entre 1848 et 1854 - poste clé mais atypique -, architecte en chef de la $1^{\text {re }}$ section du service des beaux-arts de la Ville de Paris de 1848 à 1860, architecte en chef de l'Hôtel de Ville de Paris à partir de 1850, enfin, consécration suprême, directeur du service d'architecture de la Ville de Paris entre 1860 et 1870 .

10 Ces multiples responsabilités ont généré, au premier chef, une abondante production écrite - correspondance, rapports, imprimés ou non, notes, etc. -, mais aussi, à un moindre degré dans le fonds d'archives, un certain nombre de dessins là encore réunis sous la forme de pièces individualisées. C'est ainsi que l'on relève la présence d'une série fort intéressante, mais très endommagée par les outrages du temps, de dessins aquarellés concernant des aménagements décoratifs et mobiliers projetés pour les églises parisiennes, et dont les réalisations les plus célèbres demeurent les peintures d'Hippolyte Flandrin pour Saint-Germain-des-Prés et Saint-Séverin. On note ainsi des propositions de Baltard pour la décoration de la chapelle des Fonts de cette dernière église (ill. 4), mais aussi des projets plus spécifiquement mobiliers, comme un dessin pour le maître-autel de l'église Saint-Eustache. 
III. 4 : étude, par V. Baltard, pour la décoration picturale de la chapelle des Fonts en l'église SaintSéverin,

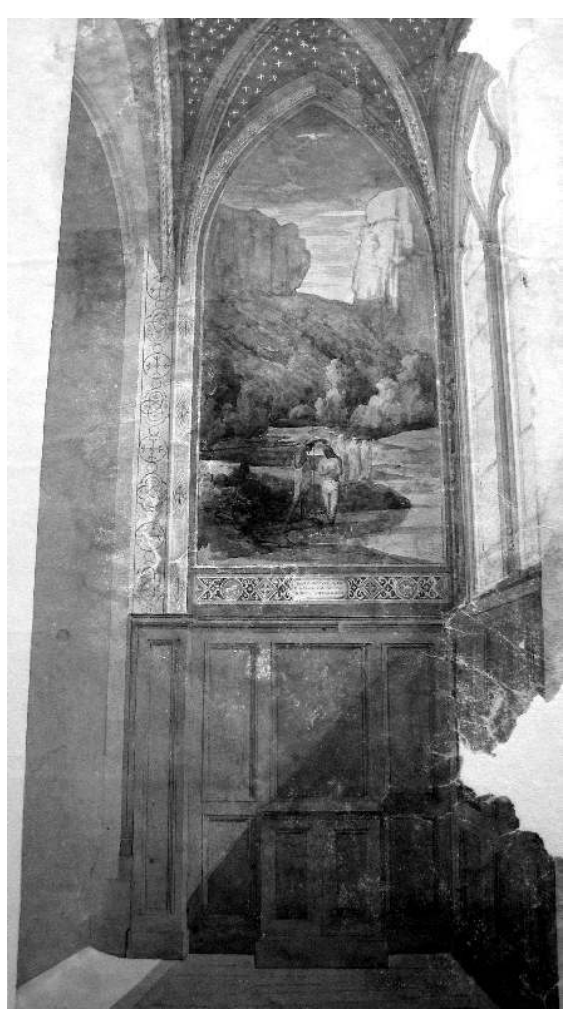

Exécutée par Paul Flandrin, 2 mai 184[7]. Arch. nat., 332/AP/26/B

Cl. de l'auteur.

11 En revanche, le plus important et célèbre programme de Baltard relatif à l'architecture religieuse parisienne, en l'occurrence la construction de l'église Saint-Augustin, au début des années 1860, n'est aucunement documenté. Seule, une série de croquis sur calque représentant différents projets d'églises peut laisser supposer que certains correspondent à des idées ou études de l'architecte pour Saint-Augustin.

En revanche, certains projets de Victor sont fort heureusement plus prolixes en archives, essentiellement écrites, ou figurées. Le plus célèbre, et qui a construit la renommée universelle de Baltard, est évidemment le projet - ou plutôt les projets - pour les Halles centrales. Il est généreusement représenté par une pléthore de pièces écrites, rapports manuscrits ou imprimés - en grand nombre, notes, correspondance, s'échelonnant de 1842 jusqu'à la mort de Baltard, et même au-delà avec, en particulier, un dossier se rapportant à la dénomination de Baltard donnée à une partie de l'ancienne rue du PontNeuf, ou encore quelques pièces beaucoup plus tardives, insérées par les descendants de l'architecte, et qui concernent les contestations relatives à la paternité de Baltard sur la réalisation définitive des Halles. Les enquêtes sur la construction des marchés en France et à l'étranger au milieu du XIX ${ }^{\mathrm{e}}$ siècle ou encore les projets concurrents de celui de Baltard et Callet, approuvé en 1851 et 1853, et qui ont comme auteurs principaux Hector Horeau ou Charles Duval, sont également bien documentés. Curieusement, le parent pauvre demeure, là encore, les documents figurés avec un petit nombre de plans, la plupart imprimés et joints aux rapports écrits, quelques croquis ou esquisses manuscrits et une seule photographie (ill. 5). 


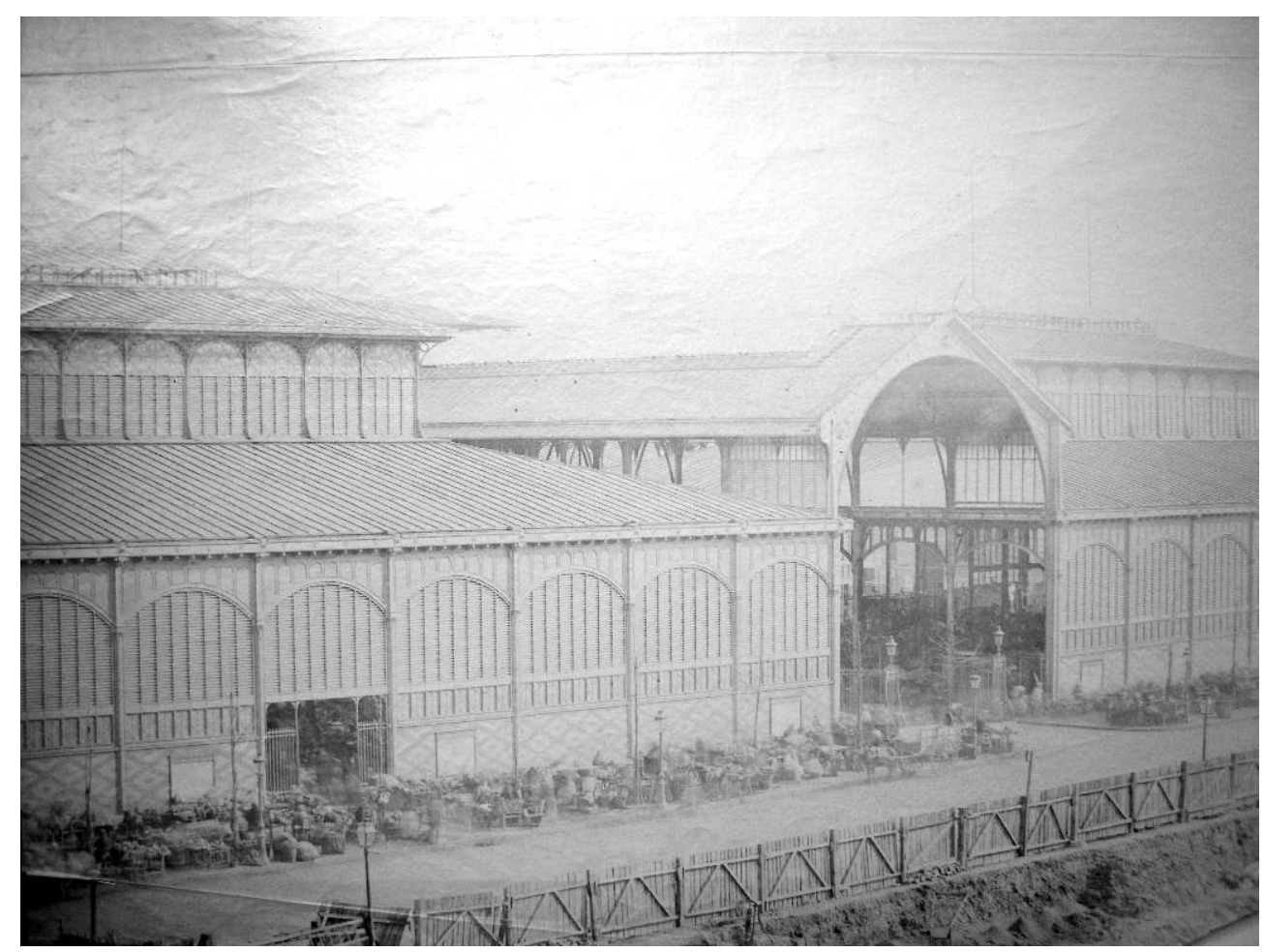

[vers 1860]. Arch. nat., 332/AP/26/E

Cl. de l'auteur.

Les photographies sont à l'inverse plus nombreuses pour ce qui a trait aux ouvrages d'embellissement ou de décoration de l'ancien Hôtel de Ville de Paris, dirigés par Baltard, entre autres les diverses fêtes et réceptions qui y furent données, la construction de l'escalier en fer à cheval de la cour intérieure, l'ornementation des salles de réception et, après l'incendie de 1870-1871, les pièces écrites relatives au concours organisé pour la reconstruction-restauration de l'Hôtel de Ville, pour lequel Baltard eut l'extrême désappointement de ne pas voir son projet de restauration «à l'identique " retenu, en 1873, au bénéfice du projet de reconstruction de Théodore Ballu.

Quelques réalisations de faible importance architecturale sont également disséminées au fil des pièces isolées (ainsi un projet de lavoir pour la commune de Vauhallan, dans l'Essonne), ainsi que plusieurs interventions menées en collaboration avec d'autres architectes : avec Gustave Huillard, élève et principal collaborateur de Baltard sous le Second Empire pour le marché du Temple; avec le bordelais Henri Duphot, pour la construction et aménagement du château de Cestas, propriété du baron Haussmann.

En résumé, ce fonds, particulièrement disparate et hétérogène, se caractérise par le volume abondant de ses pièces écrites, souvent regroupées de manière cohérente, alors que la partie iconographique, fort malmenée et ayant souffert d'anciennes conditions de conservation déplorables, se présente sous la forme d'une collection de pièces individualisées ayant le plus souvent perdu tout lien avec leur contexte de production écrit. 


\section{Les archives de la dynastie Destailleur : François- Hippolyte (1787-1852), Hippolyte-Alexandre (1822-1893) et Walter-André (1867-1940)}

Si le fonds Baltard concerne principalement, en raison du parcours professionnel de ses acteurs, la sphère publique, le fonds Destailleur ne se rapporte presque exclusivement qu'à la sphère de la commande privée. D'où une différence notable d'appréciation dans la valeur documentaire de ces sources. Si l'absence presque totale de pièces se rapportant, par exemple, aux travaux du Panthéon dirigés par Louis-Pierre Baltard, peut être compensée par d'autres sources administratives (sous-séries $\mathrm{F}^{13}, \mathrm{~F}^{21}$ ou AJ ${ }^{56}$ des Archives nationales), rien, en revanche, ne peut suppléer - si ce n'est d'improbables archives de commanditaires - l'absence des dossiers de projets relatifs à la commande privée. Il en résulte que le fonds Destailleur revêt une richesse documentaire primordiale, à divers titres.

17 Ce sont tout d'abord la personnalité et le renom de Hippolyte-Alexandre, architecte célébré par la société du Second Empire et des débuts de la Troisième République et qui occupe, pendant une longue période, une position quasi-monopolistique auprès des commanditaires issus de l'aristocratie et de la haute bourgeoisie. Ce succès considérable, lié aussi bien à son expertise et sa pratique du style rocaille, très en vogue auprès de la société impériale, qu'à ses talents propres d'architecte-restaurateur d'édifices anciens qu'il adapte au goût néo-Renaissance, néo-Rocaille, en passant par l'influence du premier $\mathrm{XVII}^{\mathrm{e}}$ siècle français, lui vaudra même - tout autant que ses liens familiaux en Angleterre - la faveur de l'ex-impératrice Eugénie, qui lui confiera les travaux de réaménagement de son domaine de Farnborough.

Ce sont ensuite les qualités intrinsèques et la teneur même de ce fonds d'archives, entré aux Archives nationales en 1989, après avoir transité par le centre de documentation sur l'histoire de la construction du conservatoire national des arts et métiers, qui lui confèrent le plus haut intérêt. En effet, même si la quasi-totalité des pièces écrites n'est pas parvenue jusqu'à nous, le fonds est divisé en dossiers de projets souvent particulièrement complets en ce qui concerne les documents figurés et qui illustrent l'ensemble du processus opérationnel, depuis les esquisses et croquis préparatoires jusqu'aux dessins de présentation soigneusement lavés. On peut par conséquent suivre avec une relative précision les différentes phases d'un projet, et, en particulier, par les dessins d'exécution, son déroulement concret, par les nombreux détails grandeur d'exécution et attachements qui émaillent les dossiers. Les difficultés d'analyse des plans résultent essentiellement de l'absence de pièces écrites qui pourraient éclairer les variantes d'un projet, mais aussi de la disparition d'une part notable de l'œuvre construite, qui en renforce a contrario la valeur documentaire intrinsèque.

On ne fera qu'effleurer la personnalité de François-Hippolyte Destailleur, architecte du ministère des finances et, surtout, d'une éclatante clientèle privée, dont la production graphique n'est documentée dans ce fonds que par quelques pièces éparses (deux dessins d'exécution de l'hôtel de Noailles, rue d'Astorg, datés de 1836, entre autres). On s'attardera un peu plus longuement sur Hippolyte-Alexandre (ill. 6), dont l'œuvre est représentée par environ la moitié des portefeuilles composant les papiers Destailleur. 


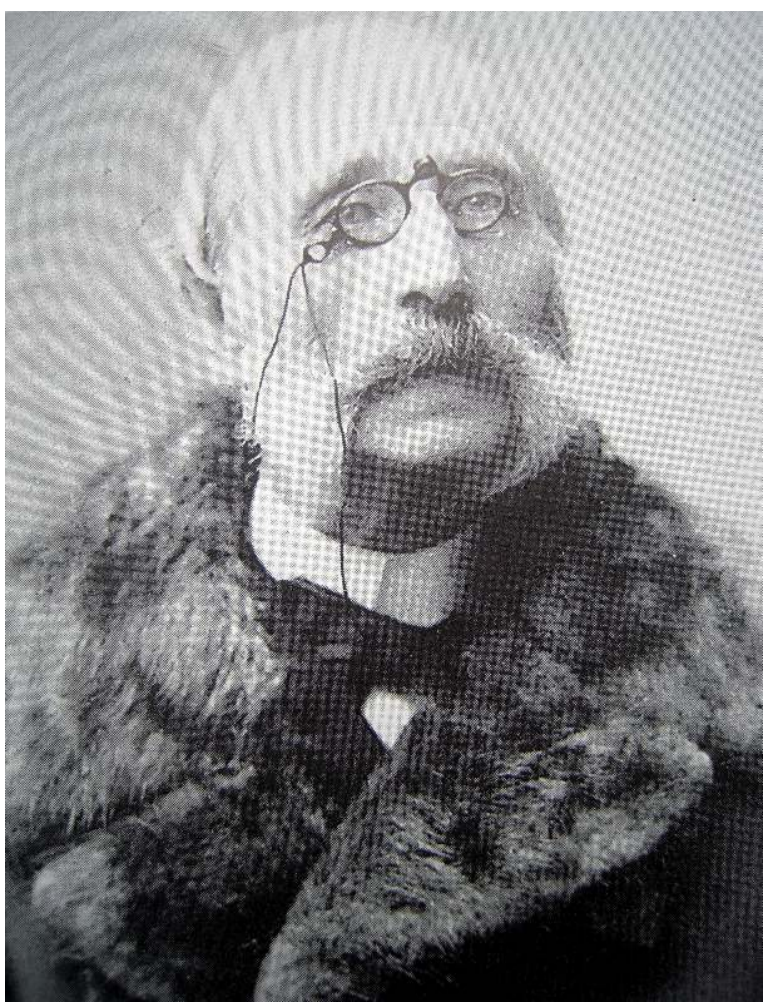

Épreuve photographique anonyme non datée, [vers 1885]. Archives de l'Académie d'architecture, fonds de la Société centrale des architectes (photographie reproduite dans Gérard Rousset-Charny, Les Palais parisiens de la Belle Époque, Paris, délégation à l'action artistique de la Ville de Paris, 1990, p. 38)

$\mathrm{Cl}$. de l'auteur.

Si Hippolyte-Alexandre a exercé, tout comme son père, les fonctions d'architecte du Gouvernement, au ministère de la justice et à l'hôtel de la Monnaie, les archives conservées ne se rapportent qu'à sa prolixe œuvre privée. Il acquiert sa célébrité par la restauration et le remodelage de nombreux et prestigieux domaines, au premier rang desquels il faut mentionner les châteaux de Courances et de Vaux-le-Vicomte (ce dernier lacunaire dans les papiers Destailleur), mais on peut également citer les châteaux de Maintenon (Eure-et-Loir) et de Mouchy-le-Châtel (Oise), deux propriétés des Noailles, dont les Destailleur sont les architectes attitrés tout au long du XIX ${ }^{\mathrm{e}}$ siècle. Dans le cas de Mouchy-le-Châtel, d'autant plus intéressant qu'il a aujourd'hui disparu, Destailleur procède, dès les années 1850 , et principalement entre 1866 et 1870 , à un rhabillage systématique des façades sur la cour et sur le jardin dans le goût néo-Renaissance. De nombreux relevés de "l'état actuel» et propositions de restauration témoignent des instructions des commanditaires et des modèles auxquels fait référence le jeune architecte. Comme dans la plupart des dossiers de projets, on y relève de nombreux dessins grandeur d'exécution, que ce soit pour le profil des moulures de baies ou pour la restitution de créneaux et mâchicoulis de tour (ill. 7). 
III. 7 : projet de restauration et reconstruction du château de Mouchy-le-Châtel (Oise) :

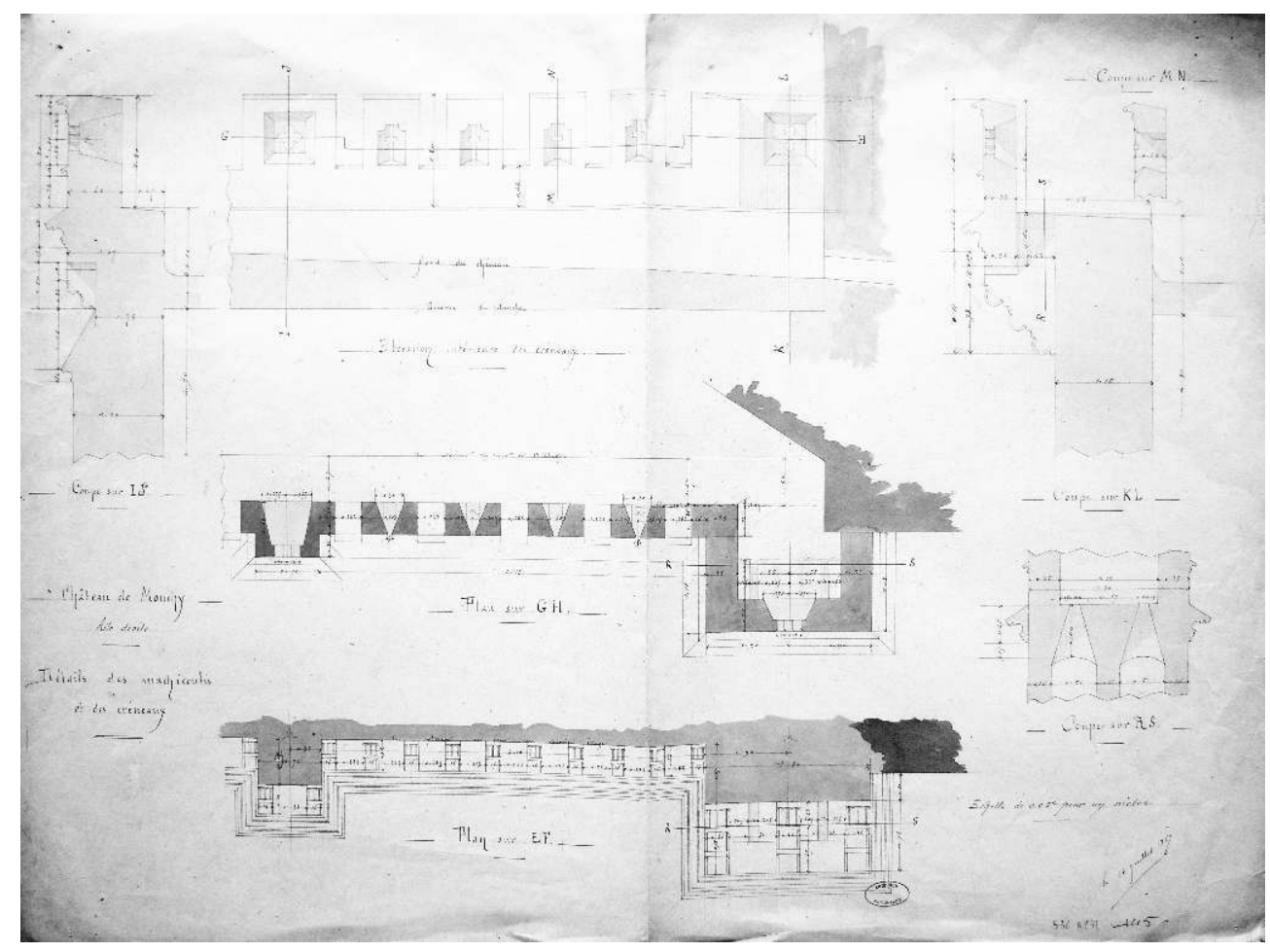

Détails des mâchicoulis et des créneaux de l'aile droite (par H.-Al. Destailleur, 16 juillet 1867). Arch. nat., 536/AP/48

$\mathrm{Cl}$. de l'auteur.

Intervenant également pour le dessin d'ornementation intérieure et de mobilier, Destailleur fait montre d'une grande efficacité et d'un rythme soutenu dans la production de propositions et de variantes.

Hippolyte-Alexandre n'intervient pas uniquement sur des édifices existants, se rapportant le plus souvent aux XVI ${ }^{e}, X_{I I}$ ou XVIII ${ }^{e}$ siècles. Il fait également œuvre d'architecte constructeur, comme l'atteste, parmi d'autres exemples, celui du château de Franconville à Saint-Martin-du-Tertre, élevé en 1876 pour le duc de Massa (ill. 8). 


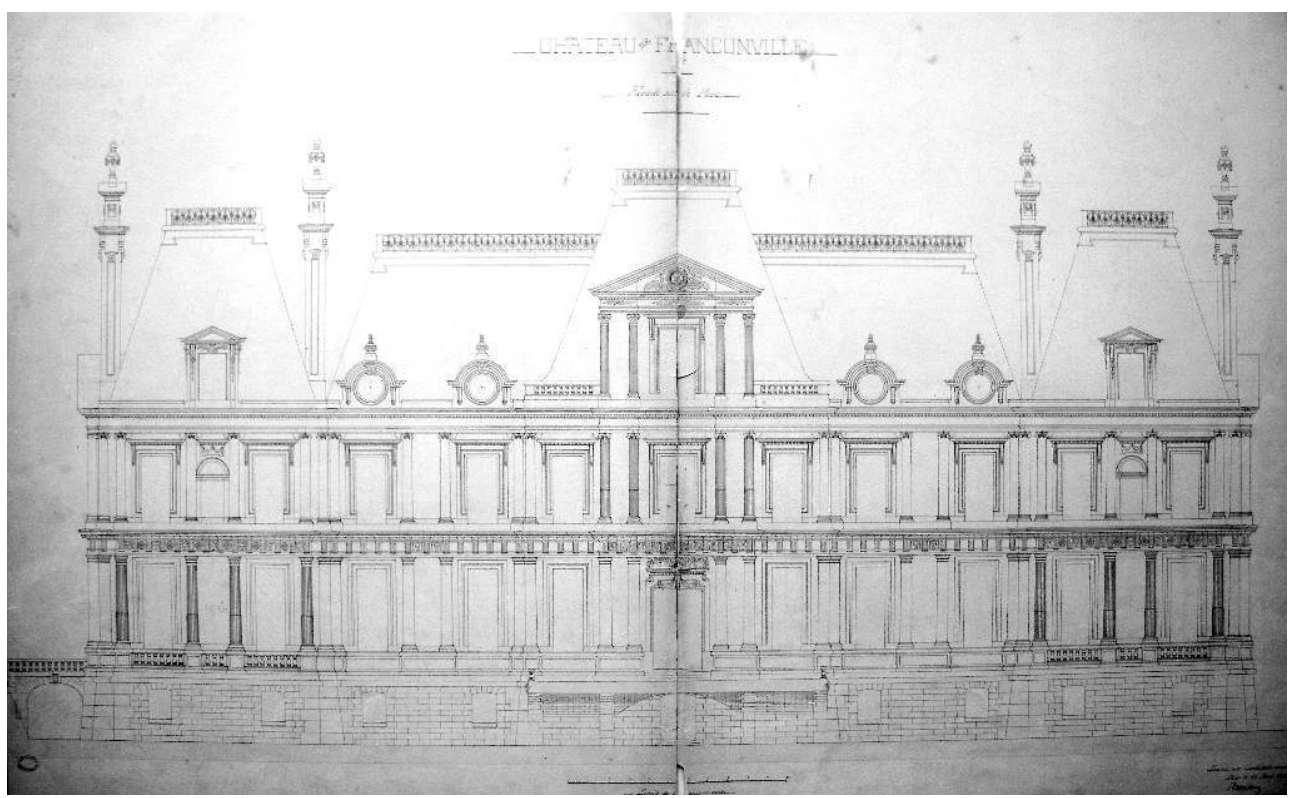

Par H.-Al. Destailleur, 22 avril 1876. Arch. nat., 536/AP/92

$\mathrm{Cl}$. de l'auteur.

La façade sur cour de ce château fait référence explicite au modèle, vénéré par Destailleur, du château de Maisons. Rappelons que, par les collections de dessins anciens qu'il a réunies, et qui ont fait tout autant la célébrité de son nom que ses propres réalisations, l'architecte dispose d'un répertoire formel d'une grande variété où il peut puiser très souvent modèles ou références.

Destailleur réaménage profondément ou construit en parallèle à Paris de nombreux hôtels particuliers pour sa clientèle fortunée. Si les deux hôtels de Béhague, avenue Bosquet, construits pour la comtesse de Béhague et son fils Octave, en 1866-1868, en sont l'une des plus célèbres opérations, on peut relever les exemples de l'hôtel élevé pour le duc de Conegliano, rue de Ponthieu, en 1888-1889 ou, à un degré plus «modeste », l'hôtel Viollier, rue Rembrandt, en 1878. Dans la plupart des cas, les dossiers se composent de plans, coupes et élévations de l'état ultime, bon pour exécution, mais aussi de nombreux détails grandeur se rapportant aussi bien au gros œuvre qu'aux aménagements ornementaux.

Hippolyte-Alexandre intervient essentiellement dans les typologies d'immeubles privés (châteaux, hôtels particuliers), mais on lui doit aussi de rares commandes extérieures, comme celle de la chapelle des catéchismes de l'église Sainte-Clotilde à Paris, en 1878.

Walter-André, âgé de 26 ans à la mort de son père, suit néanmoins d'emblée et avec ardeur le chemin ouvert par celui-ci et parvient à conserver - contrairement, semble-t-il, au fils Sanson, décédé certes prématurément - la confiance d'une clientèle aussi exigeante que versatile. Pour le comte de Béarn, héritier des Béhague, il poursuit, à partir de 1895, l'aménagement et l'agrandissement de l'hôtel de l'avenue Bosquet, en l'ouvrant sur la rue Saint-Dominique, et en n'hésitant pas, au passage, à sacrifier le grand hôtel construit par son père... Il fait appel, tout comme son père, aux références du classicisme français, en s'inspirant de l'escalier de la Reine à Versailles, pour concevoir le décor en marbre polychrome du grand escalier du nouvel hôtel de Béarn (ill. 9) 
III. 9 : Projet de décoration en marbre polychrome pour le grand escalier de l'hôtel de Béarn, rue Saint-Dominique et avenue Bosquet

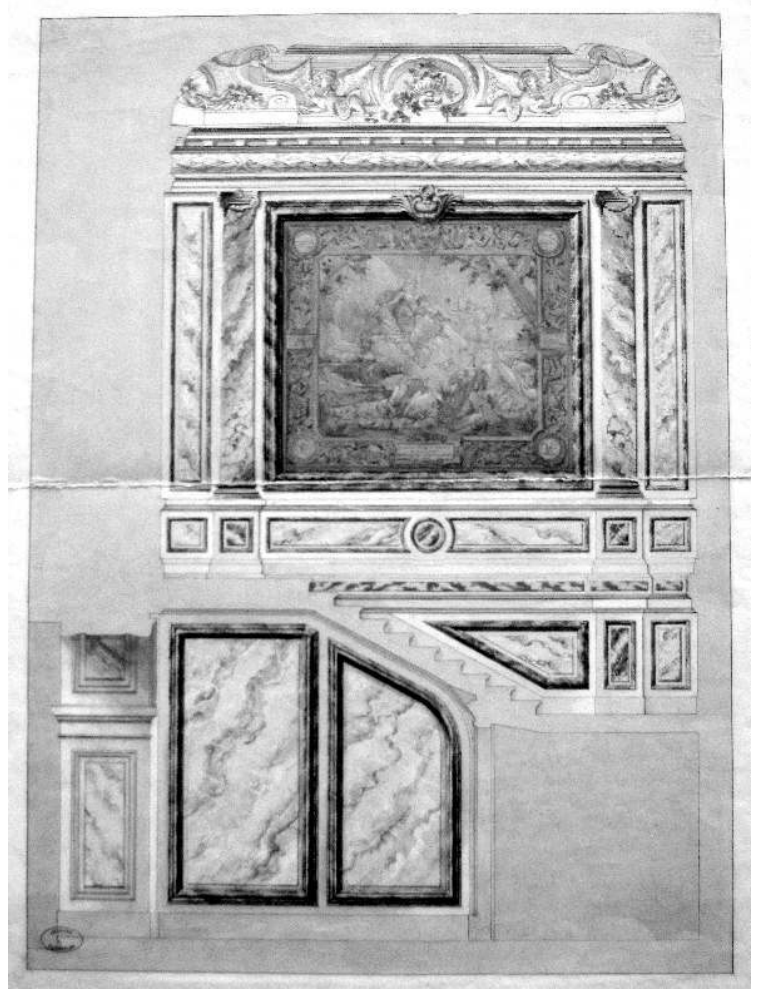

Par Walter-André Destailleur, s.d. Arch. nat., 536/AP/2

Cl. de l'auteur.

27 C'est au second époux de la comtesse de Béhague, James de Kerjagu, que Walter-André doit, entre 1893 et 1898, l'une de ses plus importantes commandes, celle du château de Trévarez (Finistère) où, là encore, l'architecte fait emploi des références paternelles en composant un édifice en brique et pierre directement inspiré de la première Renaissance française (ill. 10). 


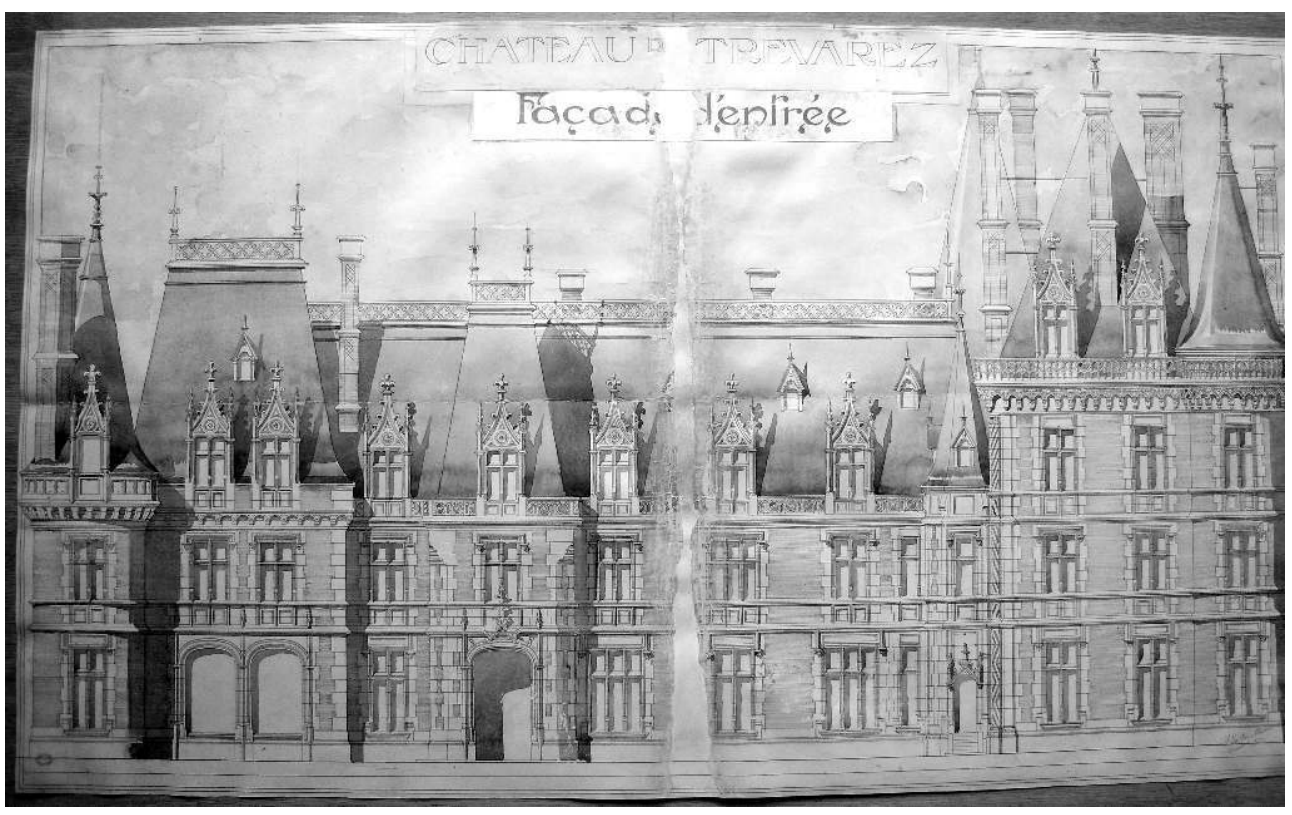

Par W.-A. Destailleur, novembre 1893. Arch. nat., 536/AP/87

Cl. de l'auteur.

Mais il développe, augmente et diversifie également sa clientèle et les programmes qui lui sont confiés. Le banquier Louis Cahen d'Anvers lui accorde en l'occurrence un puissant soutien, en le chargeant, dès 1895 , de la complète et délicate restauration du château de Champs, qu'il vient d'acquérir, alors que ce sont Henri et Achille Duchêne qui reçoivent la mission de reconstituer le parc régulier. Outre la remise en état du château, Destailleur construit ou reconstruit de nombreuses dépendances: orangerie, écuries, maison du jardinier. Il intervient pour le même commanditaire, que ce soit en son hôtel de la rue de Bassano, au château d'Abondant (Eure-et-Loir) et même pour un chalet à Gérardmer. Dans la composition même des dossiers de Walter-André, la part représentée par les plans d'entreprises est croissante, probablement encouragée par le développement rapide des procédés industriels de tirages de calques. En revanche, la part des dessins manuscrits préparatoires tend à se raréfier au regard des dossiers paternels. Dans la première moitié du XXe siècle, la production de l'architecte connait un rythme qui se diversifie, avec des appropriations d'immeubles à usage industriel ou commercial (garage Renault, avenue des Champs-Élysées), la construction d'immeubles de rapport ou des interventions sur les chantiers de la Première Reconstruction (Limé et Belleau dans l'Aisne).

La richesse du fonds Destailleur résulte d'une conservation souvent remarquable des dossiers de documents figurés, qui ont pu même conserver leur conditionnement originel en certains cas. Il éclaire ainsi de manière souvent extrêmement précise le déroulement des chantiers dans leurs multiples composantes et péripéties, pour une période peu ou insuffisamment documentée pour la commande privée d'architecture résidentielle. Les dossiers ont également souvent conservé l'intégralité de leurs subdivisions se rapportant aux dépendances des domaines où l'architecte a été amené à intervenir, que ce soit les communs, les fermes, voire les chapelles castrales ou sépulcrales.

Cette homogénéité des dossiers permet ainsi au chercheur de disposer d'une appréciation globale sur la teneur des interventions de l'architecte pour tel ou tel commanditaire. 
Même si d'autres éléments importants des archives Destailleur sont conservés, en France, à l'Académie d'architecture, et en Allemagne, à la Kunstbibliothek de Berlin, les papiers d'agence, tels qu'ils sont parvenus aux Archives nationales, livrent un éclairage très complet sur la commande privée en France, dans la seconde moitié du XIX ${ }^{e}$ siècle.

En dépit de l'extrême disparité de leur contenu et de la production dont ils illustrent l'élaboration, les fonds privés d'architectes révèlent l'œuvre d'art totale que constitue, au $\mathrm{XIX}^{\mathrm{e}}$ siècle, mais aussi dans la première moitié $\mathrm{du} \mathrm{XX}^{\mathrm{e}}$ siècle, la création architecturale. Avant d'être un constructeur, l'architecte est un créateur, qui dessine aussi bien les formes de l'immeuble que celles de son ornementation et de son mobilier, ou encore des jardins qui l'environnent. Bien loin des préoccupations d'aménagement sériel ou de construction de masse de la fin du XX $\mathrm{XX}^{\mathrm{e}}$ siècle, les Baltard, comme les Destailleur, selon des processus de commande ou de maîtrise d'œuvre très différents, se rattachent tous à la réalisation d'un ouvrage individualisé qui s'inscrit en premier lieu dans la pensée graphique. Si les préoccupations urbanistiques sont puissamment intégrées par Victor Baltard, si le caractère fonctionnel du programme est résolument affirmé par celui-ci, notamment dans ses études de marchés, la réalisation matérielle est dictée par l'élaboration du projet qui engendre une abondante production graphique, dont les fonds des Archives nationales témoignent avec éclat. Production dont les élévations et perspectives aquarellées de l'auteur des Halles centrales ou de Walter Destailleur traduisent la volonté affichée, d'un siècle à l'autre, par ces architectes, de considérer la démarche esthétique comme consubstantielle de la pensée architecturale.

\section{RÉSUMÉS}

Les vingt-cinq fonds privés d'architectes conservés aux Archives nationales à Paris revêtent la plus grande diversité en termes de contenu, de supports et d'ampleur, et nécessitent une approche préalable spécifique de la part de la recherche. À l'inverse des versements administratifs qui s'appuient sur l'histoire et l'évolution institutionnelles, les archives privées d'architectes sont souvent le fruit de sédimentations aléatoires, illustrant aussi bien une histoire familiale que les circonvolutions de parcours professionnels recouvrant parfois plusieurs générations. Tel est le cas des «dynasties» Baltard et Destailleur, dont l'hétérogénéité des archives respectives témoignent de l'extrême variété des appréciations portées par les architectes - et leurs descendants - sur leurs propres papiers. D'un fonds mutilé et limité aux seuls dessins d'agence, pour les Destailleur, jusqu'au "magma » des archives Baltard mêlant documents écrits à caractère strictement familial, dossiers de projet et pièces isolées individualisées selon de simples critères esthétiques, la palette est large des ressources que prodiguent au chercheur ces fonds privés dont la prise en compte par les institutions publiques ne remonte guère à plus d'un demi-siècle.

The 25 private architects funds stored in the Archives nationales in Paris are, in terms of content, media and extent, of the utmost diversity, and require from the researcher a very specific preliminary approach. Contrary to administrative transfers, that are the result of institutional history and changes, private architects archives are often a product of random sedimentations, usually illustrating as much a family history as the turns of career paths going sometimes over 
several generations. Baltard and Destailleur "dynasties" are examples for such a situation. The heterogeneity of their respective archives testifies to the extreme variety of assessments made by architects - and their descendants- on documents and papers these archives were made of. From a mutilated fund, that is only made of agency drawings, for Destailleurs, to the "jumble" of Baltard archives that combined written documents strictly of a private nature, project files and isolated single pieces sorted by simple aesthetic criteria, those private funds, which only fifty years ago weren't acknowledged by public institutions, offer the researcher resources covering a very wide range.

Durch die 25 Privatnachlässe von Architekten, die von den Archives nationales in Paris aufgehoben werden, lässt sich eine ganz besondere Verschiedenheit aufweisen, was Inhalt, Form und Umfang betrifft. Im Gegenteil zu der amtlichen Archivsammlung, die sich auf Geschichte und Veränderungen des institutionellen Lebens beziehen, sind die Privatarchive der Architekten das Ergebnis von zufallsbedingten Schichten, die ebenso von dem Familienleben zeugen wie von den Umwandlungen der beruflichen Laufbahnen, über manchmal mehrere Generationen von richtigen Dynastien hinweg am Beispiel der Baltards und Destailleurs. Die uneinheitliche Auswahl beider Nachlässe zeugt von der unterschiedlichen Wertschätzung, die die Architekten und deren Nachfahren - ihren eigenen Unterlagen entgegen brachten: Einerseits der verstümmelte auf die Bauzeichnungen der Agentur begrenzte Nachlass Detailleurs, andererseits die ungeheure Mannigfaltigkeit im Nachlass von Baltard, in welchem Familienunterlagen, Bauprojektunterlagen und isolierte einfach nach ästhetischen Kriterien aufgehobene Stücke beieinander liegen. Diese Privatarchive, die erst seit einem halben Jahrhundert von der öffentlichen Archivverwaltung berücksichtigt wurden, vermitteln dem Forscher eine vielseitige Dokumentation.

\section{AUTEUR}

\section{JEAN-CHARLES CAPPRONNIER}

Jean-Charles Cappronnier, né en 1960, est docteur en histoire de l'architecture avec une thèse intitulée « L'agence d'architecture de Charles Duval et Emmanuel Gonse et les enjeux de la Première Reconstruction, 1906-1937 » (université de Versailles - Saint-Quentin-en-Yvelines, 2007). Ses problématiques de recherche portent donc sur l'architecture de la Première Reconstruction ainsi que sur l'art sacré en France au XX $\mathrm{XX}^{\mathrm{e}}$ s. et il a, à ce titre, participé entre autres, aux ouvrages Reconstructions en Picardie après 1918 (R.M.N., 2000) et La Grande Reconstruction. Reconstruire le Pas-de-Calais après la Grande Guerre (Archives départementales du Pas-de-Calais, 2002). Chargé d'études documentaires aux Archives nationales, il y est responsable du traitement et du classement des archives privées d'architectes au sein de la section des cartes, plans et de photographies. Adresse électronique : jean-charles.cappronnier@culture.gouv.fr 